\title{
Influence of intercropping systems and foliar spray by various growth stimulators on production and profitability of bed planted wheat
}

\author{
W.A. Hamd-Alla ${ }^{1}$ and T. Singh ${ }^{2}$ \\ ${ }^{I}$ Department of Crop Intensification Research, Field Crops Research Institute, Agricultural Research \\ Center, Giza, Egypt. \\ ${ }^{2}$ Department of Agronomy, Punjab Agricultural University, Ludhiana 141 004, Punjab, India.
}

Received: 10 Oct. 2019 / Accepted 15 Dec. 2019 / Publication date: 30 Dec. 2019

\begin{abstract}
A field experiment was carried out during Rabi seasons of 2017-18 and 2018-19 at the research farm of the Department of Agronomy, Punjab Agricultural University, Ludhiana, India. In order to study the effect of intercropping systems and foliar spray by various growth stimulators on production and profitability of bed planted wheat. The experiment was carried out in a randomized complete block design using spilt block arrangement with three replications. The first variable was the foliar sprays which occupied horizontally (control, salicylic acid@ 200 ppm, ascorbic acid @ 200 ppm and the combination of salicylic \& ascorbic acids @ 200 ppm). Intercropping system treatments as a second variable consisted of sole wheat, sole canola, sole linseed, sole oats fodder, wheat + canola, wheat + linseed and wheat + oats fodder, which allocated vertically. Results showed that: wheat + oats fodder seasons as compared to wheat + linseed and wheat + canola intercropping systems. The salicylic acid@ @200 ppm produced the maximum values for all the traits as compared to control in the two growing seasons. Land equivalent ratio indicated the yield advantage over sole crops. Higher values of competitive ratio of wheat showed that it was the dominant species over the intercrops in the intercropping systems. Monetary advantage index values showed the economic advantage of salicylic acid@200 with wheat + oats fodder as compared to other treatments. All the component crops (oats fodder, canola and linseed) in intercropping system with wheat resulted in the higher values of wheat equivalent yield than their respective sole planting except sole crop of canola in both seasons.
\end{abstract}

Keywords: Intercropping systems, Foliar spray, Wheat, Linseed, Wheat equivalent yield

\section{Introduction}

Intercropping is a type of mixed cropping, defined as an agricultural practice of cultivating two or more crops in the same filed at the same time. Intercropping systems have several benefits to the farmers such as, flexibility, profit maximization, risk minimization against total crop failure or disease, weed control, increase land use efficiency, soil conservation, improvement of soil fertility and balanced nutrition (Dhima et al., 2007). (Nagwa R. Ahmed et al., 2019) Intercropping of guar, cowpea and clitiora with maize had a significant effect on 100 grain and yield, intercropping clitoria with maize gave the highest values of grain yield whereas guar intercropping with maize gave the lowest values of grain yield. (Hamada and Hamd-Alla 2019) Plant height, number of spikes $/ \mathrm{m}^{2}$, grains number/spike and 1000grain weight were affected significantly by intercropping systems in both seasons. Wang et al., 2011 found that better development of wheat morphological traits in raised bed planting promoted the expression of the apparent "border effect" for wheat on the raised beds and optimized the compensation of the yield components to increase wheat grain yield. Pandey et al., 2015 reported that bed planting method improved water distribution and efficiency, fertilizer use efficiency (FUE), reduced weed infestation, crop lodging and reduced seed rate without sacrificing yield. Growing wheat in a bed planting pattern could save as much as 30-50 per cent of applied irrigation water and increased the grain yield by more than 10 per cent as compared with conventional planting method. It also improved the soil physicochemical characters, reduced the bulk density, improved the soil porosity, promotes the wheat root growth and increased the wheat yield significantly. In raised bed planted wheat, there is possibility of growing crops like oats fodder, canola and linseed in furrow. (Hussain et al., 2002) Wheat is the most important cereal crop as it is the staple food of about one-third of the world's population. Salicylic acid is a phenolic derivative, distributed in a wide range of plant species and is known to have diverse regulatory roles in the metabolism of

Corresponding Author: W.A. Hamd-Alla, Department of Crop Intensification Research, Field Crops Research Institute, Agricultural Research Center, Giza, Egypt. E-mail: bwael20@gmail.com 
plants. It is a natural product of phenylpropanoid metabolism Produced by decarboxylation of transcinnamic acid to benzoic acid and its subsequent 2- hydroxylation. It undergoes metabolism by conjugating with glucose to salicylic acid glucoside and an ester (Popova et al., 1997). Salicylic acid has direct involvement in plant growth thermogenesis, flower induction and uptake of ions. It affects ethylene biosynthesis, stomatal movement and reverses the effects of abscicic acid (ABA) on leaf abscission. On the other hand, enhancement of the level of chlorophyll and carotenoid pigments, photosynthetic rate and modifying the activity of some of the important enzymes are other roles assigned to salicylic acid (Galal et al., 2000, Abdel Ati et al., 2000). Furthermore, salicylic acid can inhibit the proteinase inhibitor synthesis induced by wounding (Doaeres et al., 1995) antioxidants acts as a signal in plant-microbe interaction and added that which are considered safe to human and environment had been used successfully to control some plant diseases (Elad 1992). Said and HamdAlla, 2018 mentioned that the highest values of yields and its attributes of maize and soybean were observed from plants which were sprayed with salicylic acid at the rate of $200 \mathrm{ppm}$ of maize + soybean. (Fercha et al., 2011). Ascorbic acid reduced the adverse effect of salt stress on plant growth which may be due, in part, to increased leaf area, improved chlorophyll (Bakry et al., 2013). $300 \mathrm{mg}$ $\mathrm{L}^{-1}$ of ascorbic acid gave the highest values for plant height, biological, grain and straw yields. Ascorbic acid as an abundant component of plants functions as an antioxidant and an enzyme cofactor. It participates in a variety of processes, including photosynthesis, cell wall growth and cell expansion, resistance to environmental stresses and synthesis of ethylene, gibberellins, anthocyanin and hydroxyl proline (Galal et al., 2000, Smirnoff and Wheeler 2000). Hussein and Alva 2014 reported that the exogenous application of ascorbic acid improved botanical growth which may contribute to increased plant biomass and yield.

Therefore, the present study was planned with the following objectives: To study the effect of two foliar sprays, i.e. salicylic and ascorbic acid on the growth and yield of wheat and intercrops (wheat + canola, wheat + linseed and wheat + oats fodder). To evaluate the production and economic viability of bed planted wheat based intercropping systems.

\section{Materials and Methods}

\section{Description of the studied area:}

The field experiment was carried out during rabi seasons of 2017-18 and 2018-19 at the research farm of the Department of Agronomy, Punjab Agricultural University (PAU), Ludhiana, India, which is situated at $30^{\circ} 54^{\prime} \mathrm{N}$ latitude and $75^{\circ} 48^{\prime} \mathrm{E}$ longitude at a height of $247 \mathrm{~m}$ above the mean sea level. The site is characterized by subtropical and semi-arid type of climate with annual rainfall of 500-750 mm. Soil of the experimental field was loamy sand (sand $77.5 \%$, silt $9.2 \%$ and clay $13.1 \%$ ) with $\mathrm{pH} 7.2$. It was moderately fertile being low in organic carbon $(0.21 \%)$, available nitrogen $\left(63.5 \mathrm{~kg} \mathrm{ha}^{-1}\right)$, available potassium $\left(122.19 \mathrm{~kg} \mathrm{ha}^{-1}\right)$ and medium in available phosphorus $\left(19.5 \mathrm{~kg} \mathrm{ha}^{-1}\right)$.

\section{Experimental treatments and design: -}

The experiment was carried out in a randomized complete block design (RCBD) using spilt block arrangement with three replications. The first variable was the foliar sprays compounds which occupied horizontally as follows:

1- Control (water spray).

2- Ascorbic acid (AA)@ 200 ppm at 60 days after sowing (DAS) and flower initiation

3- Salicylic acid (SA) @ 200 ppm at 60 days after sowing (DAS) and flower initiation.

4- The combination of salicylic \& ascorbic acids @ 200 ppm at 60 days after sowing (DAS) and flower initiation.

The second variable was intercropping system treatments which consisted of sole wheat, sole canola, sole linseed, sole oats fodder, wheat + canola, wheat + linseed and wheat + oats fodder which were allocated vertically. 


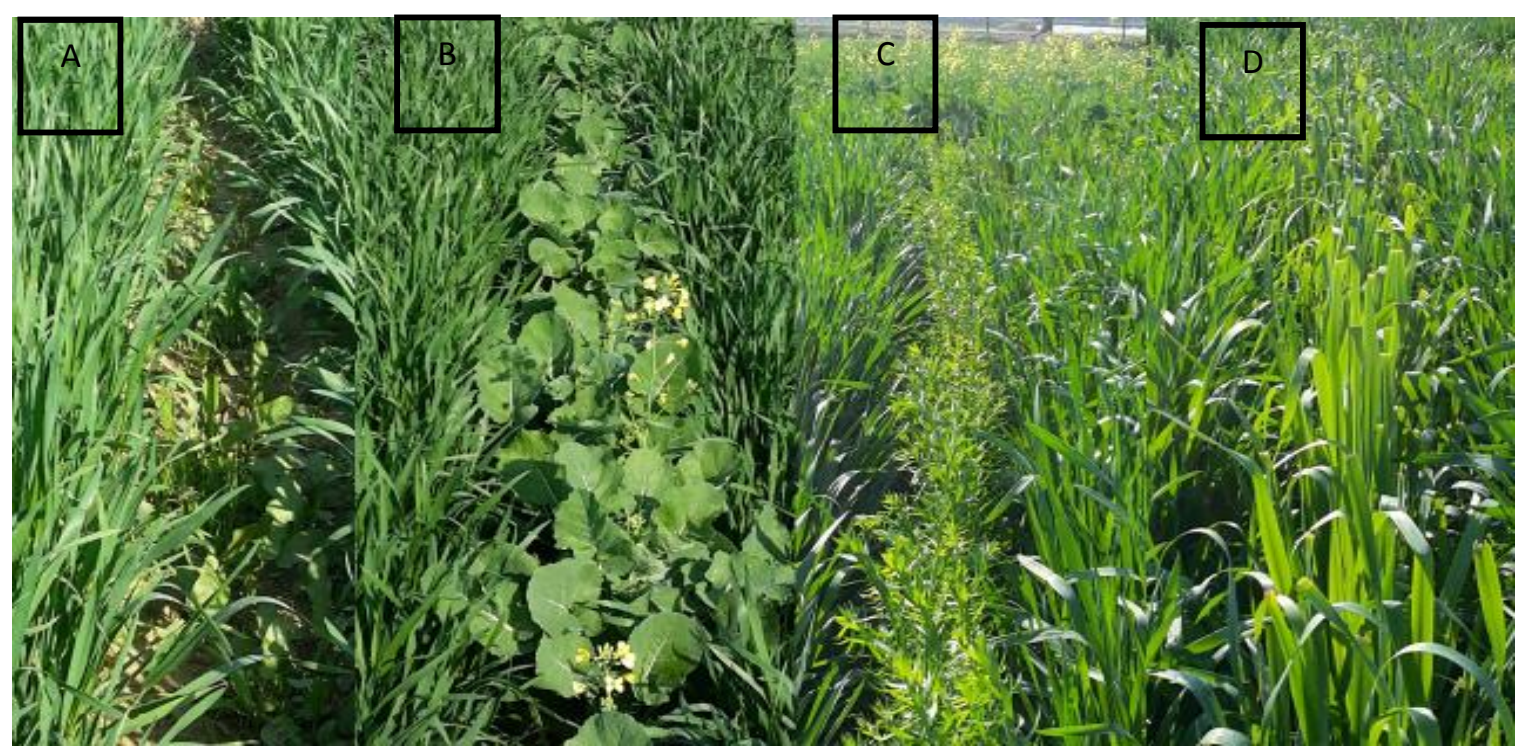

Fig. 1: Sole wheat (A); wheat + canola (B); wheat + linseed (C) and wheat + oats fodder (D).

\section{Method of sowing}

Sowing of wheat on beds was done with the help of a bed planter, which enables two wheat rows $20 \mathrm{~cm}$ apart on $37.5 \mathrm{~cm}$ wide bed and makes $30 \mathrm{~cm}$ wide furrow between two beds; and the intercrops were sown in consecutive furrows. Sowing date for the sole wheat (Triricum aestivum L.), sole canola (Brassica napus L), sole linseed (Linum usitatissimum L), sole oats fodder (Avena sativa L) and intercrops was 21 November 2017 and 10 November 2018. Wheat variety used was PBW 725 and intercrops were canola variety GSC 6, linseed variety LC2063 and oats fodder variety OL10. Wheat and intercrops were sown in 2:1 row arrangement. The recommended dose of $\mathrm{N}, \mathrm{P}$ and $\mathrm{K}$ fertilizers were applied to wheat and intercrops on area basis as given in Table 1. The control of weeds on both beds and furrow was done by hand weeding. Other package of practices for wheat and intercrops were followed as per PAU recommendations.

Table 1: The recommended doses of fertilizers applied to wheat and intercrops

\begin{tabular}{ccccc}
\hline \multirow{2}{*}{ Sr. No. } & Crop & $\mathbf{N}($ Urea) & $\begin{array}{c}\text { Recommended dose (kg/ha) } \\
\mathbf{P}_{\mathbf{2}} \mathbf{O}_{\mathbf{5}}\end{array}$ & $\mathbf{K}_{\mathbf{2}} \mathbf{O}$ \\
\hline $\mathbf{1}$ & Wheat & 125 & 62.5 & 30 \\
$\mathbf{2}$ & Canola & 100 & 30 & 15 \\
$\mathbf{3}$ & Linseed & 62.5 & 40 & - \\
$\mathbf{4}$ & Oats fodder & 75 & 20 & - \\
\hline
\end{tabular}

\section{Harvesting and threshing}

The harvesting of wheat was done manually on $14^{\text {th }}$ April, 2018 and $15^{\text {th }}$ April, 2019 The harvested crop was tied in labelled bundles and kept for sun drying. After complete drying, the threshing was carried out. Harvesting of oats fodder was done twice at 45 and 90 DAS in both the seasons for green fodder, leaving stubble height of 6-8 $\mathrm{cm}$ from ground for uniform and quick growth of the crop. The harvesting of canola was done on $27^{\text {th }}$ March 2018 and $25^{\text {th }}$ March 2019. The harvesting of linseed was done on $13^{\text {th }}$ April, 2018 and $14^{\text {th }}$ April, 2019 when the pods turned yellow and brown in colour, respectively. The harvested crop was tied in labelled bundles and kept for sun drying and then, the threshing was carried out.

\section{Observations recorded for wheat Growth parameters of wheat Leaf area}

Leaf area of the plant samples was recorded at 120 DAS using leaf area meter. The leaves of selected plants were detached and leaf area was taken with the help of Leaf Area Meter program in 
the computer by scanning of leaves. The leaf area index (LAI) was computed per plant by dividing the leaf area with land area of the plant.

$$
\text { Leaf Area Index }(\mathrm{LAI})=\frac{\text { Leaf area }\left(\mathrm{cm}^{2}\right)}{\text { Land area }\left(\mathrm{cm}^{2}\right)}
$$

\section{Photosynthetically active radiation (PAR) interception}

The Photosynthetically active radiation (PAR) interception was measured between 12:30 to 1:30 pm, on clear sunny day at monthly interval with LI-COR-LINE Quantum Sensor Photometer. The incoming and outgoing solar radiations on top of the canopy and radiation penetration at the ground surface below the crop canopy were measured. The observations were taken at random from two places in each plot. The percent interception was calculated as under:

Where,

$$
\text { PAR interception }(\text { per cent })=\frac{\operatorname{PAR}(\mathrm{I})-\mathrm{PAR}(\mathrm{T})-\mathrm{PAR}(\mathrm{R})}{\operatorname{PAR}(\mathrm{I})} \times 100
$$

PAR $(\mathrm{I})=$ Total PAR incoming above the canopy, $\mathrm{Wm}^{-2}$

PAR $(\mathrm{T})=$ PAR transmitted to ground, $\mathrm{Wm}^{-2}$

PAR $(\mathrm{R})=$ PAR reflected from the canopy, $\mathrm{Wm}^{-2}$

Chlorophyll index: Chlorophyll index was recorded at 120 DAS with the help of chlorophyll meter (Model-CCM 200).

Plant height: Five plants per plot were selected randomly to measure the height from ground level to the tip of longest leaf at harvest with a metre scale.

\section{Dry matter accumulation:}

Above ground plant samples from $50 \mathrm{~cm}$ row length were taken at maturity. The samples were first sun dried and thereafter, they were kept in oven at the temperature of $60^{\circ} \mathrm{C}$ to achieve constant weight. The dry weight thus obtained was recorded and expressed as $\left(\mathrm{g} \mathrm{m}^{-2}\right)$.

\section{Yield and yield attributing characters of wheat}

Number of spikes per meter row length: Effective tillers per meter row length from two sites in each plot were counted at harvest.

Number of grains per spike, Grain weight per spike: Five spikes were selected at random from each plot and these traits were measured.

1000 grain weight: One thousand grains from produce of each plot were taken and their weight was recorded. The thousand grain weight was expressed in grams.

\section{Biological, grain and straw yields}

Biological yield was calculated by adding grain and straw yield per plot and was reported as $\mathrm{kg}$ $\mathrm{ha}^{-1}$. The straw weight was obtained after deducting the weight of grains from total bundle weight. The total produce was weighed in bundles after harvesting and threshed, thereafter. The weight of grains was recorded. Biological straw and grain yields were computed and expressed as kg ha- ${ }^{-1}$.

\section{Observations recorded for intercrops}

Fresh forage yield for oats fodder $\left(\mathbf{k g ~ h a}^{-1}\right)$ : Two cuts were taken (either sole or intercropping) $\mathrm{I}^{\text {st }}$ at 45 DAS and II ${ }^{\text {nd }}$ at 90 DAS.

Seed yield for canola and linseed $\left(\mathrm{kg} \mathrm{ha}^{-1}\right)$ : Yield of intercrops at harvest was recorded in $\mathrm{kg} \mathrm{ha}^{-1}$

\section{Intercropping competitive indices}

The advantage of intercropping and the effect of competition between the components crops were calculated using different competitive indices. 


\section{Land equivalent ratio (LER)}

Land equivalent ratio (LER) indicates the efficiency of intercropping in using the resources of the environment compared with sole cropping (Mead and Willey 1980). This is a widely used index to assess any yield advantage in intercropping and measures the efficiency of an inter/mixed crop. Moreover, LER indicates the total land area required by sole crops to achieve the same yield as the intercrops (Willey 1985) LER was calculated as following:

$$
\mathrm{LER}=\left(\mathrm{Y}_{\mathrm{ab}} / \mathrm{Y}_{\mathrm{aa}}\right)+\left(\mathrm{Y}_{\mathrm{ba}} / \mathrm{Y}_{\mathrm{bb}}\right)
$$

Where, LER $a$ and $\operatorname{LER}_{b}$ are the partial LER of crops ' $a$ ' and ' $b$ ', respectively. $Y_{a b}$ and $Y_{b a}$ are the yields of the two component crops; $\mathrm{Y}_{\mathbf{a}}$ and $\mathrm{Y}_{\mathbf{b b}}$ are the yields per unit area when $\mathrm{a}$ and $\mathrm{b}$ grown as sole crops under those conditions with which comparison are to be made.

\section{Competitive ratio (CR)}

The competitive ratio (CR) assesses competition between component crops in intercropping system. CR gives a better measure of the competitive ability of the crops. It represents simply the ratio of individual LERs of the two component crops and takes into account the proportion of the crops in which they are initially sown (Willey and Rao 1980). The CR is calculated according to the following formula:

$$
\mathrm{CR}_{\mathrm{a}}=\left(\mathrm{LER}_{\mathrm{a}} / \mathrm{LER}_{\mathrm{b}}\right)\left(\mathrm{Zl}_{\mathrm{ba}} / \mathrm{ZI}_{\mathrm{ab}}\right) \text { while } \mathrm{CR}_{\mathrm{c}}=\left(\mathrm{LER}_{\mathrm{b}} / \mathrm{LER}_{\mathrm{a}}\right)\left(\mathrm{ZI}_{\mathrm{ab}} / \mathrm{ZI}_{\mathrm{ba}}\right) \text {. }
$$

Where, $\mathrm{LER}_{\mathrm{a}}$ and $\mathrm{LER}_{\mathrm{b}}$ are the partial land equivalent ratio of crop ' $\mathrm{a}$ ' and ' $\mathrm{b}$ '.

\section{Economic analysis}

\section{Monetary advantage index (MAI)}

However, none of the above competition indices provides any clear information on the economic advantage of the intercropping system. For this reason, the monetary advantage index (MAI) was calculated as: $\mathrm{MAI}\left(\mathrm{ha}^{-1}\right)=$

$$
\text { Commercial value of combined crops } \times \frac{\text { LER }-1}{\text { LER }} \quad \text { (Ghosh 2004) }
$$

\section{Wheat equivalent yield (WEY)}

Yield of individual crop was converted into equivalent yield (q ha-1) on the basis of prevailing market price of the crop (Anjaneyulu et al., 1982). The average prices (two years) of wheat, canola, linseed grain and straw and fresh forage yield of oats fodder for calculation of WEY and monetary advantage index (MAI) were taken as $260,593,748$ and 2 (US\$)/ton respectively. It was calculated by the following formula:

$$
\mathrm{WEY}=\text { Grain yield of wheat }+
$$

Yield of intercrops $\times$ Price of intercrops

\section{Price of wheat}

\section{Statistical analysis:}

Analysis of variance was carried out using SAS program version 9.2 (SAS, 2008). Means were compared using Least Significant Difference (LSD) at 5\% level of significant (Steel and Torrie, 1981).

\section{Results and Discussion}

\section{Effect of foliar sprays and intercropping system on yield:}

\section{I- Wheat:}

\section{Effect of intercropping systems on yield and its attributes.}

The data are presented in Table 1, 2, 3 and 4. There was a significant effect of intercropping system on yield and its attributes except straw yield in the second season. Intercropping system which were statistically at par with wheat + oats fodder significantly higher than wheat + linseed and wheat + canola intercropping systems. At 120 DAS, leaf area (2.53 and 2.49), PAR interception (72.74 and 72.64 per cent) and chlorophyll index (51.73 and 51.33) in the first and second seasons, respectively were significantly higher in wheat + oats fodder. Among the intercropping systems, higher yield 
Middle East J. Agric. Res., 8(4): 1344-1360, 2019

Table 1: Effect of intercropping systems, foliar sprays and their interaction on leaf area and PAR interception and chlorophyll index at 120 DAS of wheat.

\begin{tabular}{|c|c|c|c|c|c|c|c|c|c|c|c|c|c|c|c|}
\hline \multirow{2}{*}{$\begin{array}{l}\text { Intercropping } \\
\text { systems (I) } \\
\text { Foliar sprays } \\
\text { (F) }\end{array}$} & \multicolumn{5}{|c|}{ Leaf area } & \multicolumn{5}{|c|}{ PAR interception (per cent) } & \multicolumn{5}{|c|}{ Chlorophyll index } \\
\hline & $\begin{array}{c}\text { Sole } \\
\text { wheat }\end{array}$ & $\begin{array}{l}\text { Wheat } \\
\text { + canola }\end{array}$ & $\begin{array}{c}\text { Wheat } \\
+ \\
\text { linseed }\end{array}$ & $\begin{array}{c}\text { Wheat } \\
+ \\
\text { oats } \\
\text { fodder }\end{array}$ & Mean & $\begin{array}{c}\text { Sole } \\
\text { whea } \\
\text { t }\end{array}$ & $\begin{array}{c}\text { Wheat } \\
+ \\
\text { canola }\end{array}$ & $\begin{array}{c}\text { Wheat } \\
+ \\
\text { linseed }\end{array}$ & $\begin{array}{c}\text { Wheat } \\
+ \\
\text { oats } \\
\text { fodder }\end{array}$ & Mean & $\begin{array}{c}\text { Sole } \\
\text { wheat }\end{array}$ & $\begin{array}{c}\text { Wheat } \\
+ \\
\text { canola }\end{array}$ & $\begin{array}{c}\text { Wheat } \\
+ \\
\text { linseed }\end{array}$ & $\begin{array}{c}\text { Wheat } \\
+ \\
\text { oats } \\
\text { fodder }\end{array}$ & Mean \\
\hline & \multicolumn{15}{|c|}{ Season 2017-18 } \\
\hline Control & 2.43 & 2.03 & 2.20 & 2.30 & 2.24 & 72.32 & 61.21 & 65.42 & 68.94 & 66.97 & 49.70 & 45.08 & 46.75 & 47.68 & 47.30 \\
\hline Salicylic acid & 2.90 & 2.47 & 2.66 & 2.73 & 2.69 & 78.88 & 68.83 & 72.04 & 76.36 & 74.03 & 58.11 & 52.29 & 54.41 & 56.13 & 55.24 \\
\hline Ascorbic acid & 2.63 & 2.33 & 2.53 & 2.60 & 2.52 & 77.07 & 67.18 & 72.94 & 74.32 & 72.88 & 56.49 & 49.44 & 52.71 & 54.20 & 53.21 \\
\hline $\begin{array}{l}\text { Salicylic \& } \\
\text { Ascorbic acid }\end{array}$ & 2.53 & 2.20 & 2.37 & 2.50 & 2.40 & 74.72 & 65.64 & 67.96 & 71.33 & 69.91 & 51.31 & 45.98 & 47.87 & 48.89 & 48.51 \\
\hline Mean & 2.62 & 2.26 & 2.44 & 2.53 & 2.46 & 75.75 & 65.72 & 69.59 & 72.74 & 70.95 & 53.90 & 48.20 & 50.44 & 51.73 & 51.07 \\
\hline & \multicolumn{15}{|c|}{ L.S.D (0.05) } \\
\hline $\mathbf{F}$ & & & 0.04 & & & & & 2.13 & & & & & 1.94 & & \\
\hline I & & & 0.12 & & & & & 2.35 & & & & & 1.11 & & \\
\hline \multirow[t]{2}{*}{$\mathbf{F} \times \mathbf{I}$} & \multirow{2}{*}{\multicolumn{15}{|c|}{$\begin{array}{c}\text { NS } \\
\text { Season 2018-19 }\end{array}$}} \\
\hline & & & & & & & & & & & & & & & \\
\hline Control & 2.37 & 2.00 & 2.20 & 2.27 & 2.21 & 71.95 & 60.56 & 65.41 & 69.39 & 66.83 & 48.24 & 43.52 & 46.29 & 47.58 & 46.41 \\
\hline Salicylic acid & 2.97 & 2.30 & 2.60 & 2.70 & 2.64 & 78.86 & 69.66 & 71.30 & 75.18 & 73.75 & 59.17 & 52.05 & 54.79 & 55.69 & 55.43 \\
\hline Ascorbic acid & 2.73 & 2.23 & 2.47 & 2.50 & 2.48 & 76.48 & 65.88 & 71.69 & 74.57 & 72.16 & 55.93 & 48.95 & 52.50 & 53.56 & 52.74 \\
\hline $\begin{array}{l}\text { Salicylic \& } \\
\text { Ascorbic acid }\end{array}$ & 2.57 & 2.10 & 2.30 & 2.50 & 2.37 & 74.02 & 64.10 & 66.61 & 71.41 & 69.04 & 50.77 & 45.14 & 47.26 & 48.50 & 47.92 \\
\hline Mean & 2.66 & 2.16 & 2.39 & 2.49 & 2.43 & 75.33 & 65.05 & 68.75 & 72.64 & 70.44 & 53.53 & 47.42 & 50.21 & 51.33 & 50.62 \\
\hline & \multicolumn{15}{|c|}{ L.S.D (0.05) } \\
\hline $\mathbf{F}$ & & & 0.11 & & & & & 1.77 & & & & & 2.04 & & \\
\hline I & & & 0.19 & & & & & 2.79 & & & & & 1.15 & & \\
\hline $\mathbf{F} \times \mathbf{I}$ & & & NS & & & & & NS & & & & & NS & & \\
\hline
\end{tabular}


Middle East J. Agric. Res., 8(4): 1344-1360, 2019

Table 2: Effect of intercropping systems, foliar sprays and their interaction on plant height, dry matter accumulation and number of spikes $\mathrm{m}^{-1}$ of wheat at harvest.

\begin{tabular}{|c|c|c|c|c|c|c|c|c|c|c|c|c|c|c|c|}
\hline \multirow{2}{*}{$\begin{array}{r}\text { Intercropping } \\
\text { systems (I) }\end{array}$} & \multicolumn{5}{|c|}{ Plant height (cm) } & \multicolumn{5}{|c|}{ Dry matter accumulation $\left(\mathrm{g} \mathrm{m}^{-2}\right)$} & \multicolumn{5}{|c|}{ Number of spike $\mathrm{m}^{-1}$} \\
\hline & $\begin{array}{c}\text { Sole } \\
\text { wheat }\end{array}$ & $\begin{array}{c}\text { Wheat } \\
+ \\
\text { canola }\end{array}$ & $\begin{array}{c}\text { Wheat } \\
+ \\
\text { linseed }\end{array}$ & $\begin{array}{c}\text { Wheat } \\
+ \\
\text { oats } \\
\text { fodder }\end{array}$ & Mean & $\begin{array}{c}\text { Sole } \\
\text { wheat }\end{array}$ & $\begin{array}{c}\text { Wheat } \\
+ \\
\text { canola }\end{array}$ & $\begin{array}{c}\text { Wheat } \\
+ \\
\text { linseed }\end{array}$ & $\begin{array}{c}\text { Wheat } \\
+ \\
\text { oats } \\
\text { fodder }\end{array}$ & Mean & $\begin{array}{c}\text { Sole } \\
\text { wheat }\end{array}$ & $\begin{array}{c}\text { Wheat }+ \\
\text { canola }\end{array}$ & $\begin{array}{c}\text { Wheat } \\
+ \\
\text { linseed }\end{array}$ & $\begin{array}{l}\text { Wheat } \\
\text { + oats } \\
\text { fodder }\end{array}$ & Mean \\
\hline & \multicolumn{15}{|c|}{ Season 2017-18 } \\
\hline Control & 101.33 & 101.97 & 102.93 & 103.27 & 102.38 & 1190.04 & 1035.62 & 1135.76 & 1145.80 & 1126.81 & 123.53 & 116.41 & 118.28 & 119.36 & 119.40 \\
\hline Salicylic acid & 107.27 & 107.90 & 108.20 & 108.77 & 108.04 & 1414.56 & 1188.34 & 1248.88 & 1324.26 & 1294.01 & 133.26 & 125.65 & 127.17 & 128.14 & 128.56 \\
\hline Ascorbic acid & 105.57 & 106.00 & 106.63 & 107.17 & 106.34 & 1344.42 & 1166.20 & 1228.22 & 1268.10 & 1251.74 & 130.60 & 123.92 & 125.17 & 126.27 & 126.49 \\
\hline $\begin{array}{l}\text { Salicylic \& } \\
\text { Ascorbic acid }\end{array}$ & 103.46 & 103.80 & 104.40 & 104.93 & 104.15 & 1273.40 & 1112.12 & 1177.00 & 1214.30 & 1194.21 & 128.38 & 121.59 & 123.67 & 124.76 & 124.60 \\
\hline Mean & 104.41 & 104.92 & 105.54 & 106.04 & 105.23 & 1305.61 & 1125.57 & 1197.47 & 1238.12 & 1216.69 & 128.94 & 121.89 & 123.57 & 124.63 & 124.76 \\
\hline & \multicolumn{15}{|c|}{$\begin{array}{ll}\text { L.S.D (0.05) } \\
\end{array}$} \\
\hline $\mathbf{F}$ & & \multicolumn{5}{|c|}{76.89} & \multicolumn{5}{|c|}{1.98} \\
\hline I & \multicolumn{5}{|c|}{0.54} & \multicolumn{5}{|c|}{57.35} & \multicolumn{5}{|c|}{3.54} \\
\hline $\mathbf{F} \times \mathbf{I}$ & \multicolumn{5}{|c|}{ NS } & \multicolumn{5}{|c|}{35.89} & \multirow{2}{*}{\multicolumn{5}{|c|}{ NS }} \\
\hline & \multicolumn{13}{|c|}{ Season 2018-19 } & & \\
\hline Control & 100.70 & 101.13 & 102.37 & 103.00 & 101.80 & 1187.11 & 1037.77 & 1137.00 & 1156.11 & 1129.50 & 122.67 & 116.57 & 117.67 & 119.89 & 119.20 \\
\hline Salicylic acid & 107.13 & 107.55 & 108.20 & 108.72 & 107.90 & 1408.36 & 1187.18 & 1243.26 & 1323.95 & 1290.69 & 130.88 & 126.48 & 126.97 & 128.17 & 128.13 \\
\hline Ascorbic acid & 105.50 & 105.73 & 106.27 & 106.70 & 106.05 & 1341.56 & 1163.84 & 1222.85 & 1261.25 & 1247.38 & 129.49 & 122.60 & 124.20 & 125.46 & 125.44 \\
\hline $\begin{array}{l}\text { Salicylic \& } \\
\text { Ascorbic acid }\end{array}$ & 103.60 & 103.33 & 104.10 & 104.83 & 103.97 & 1272.65 & 1109.19 & 1172.67 & 1213.14 & 1191.91 & 128.02 & 120.53 & 122.70 & 124.17 & 123.86 \\
\hline \multirow[t]{2}{*}{ Mean } & 104.23 & 104.44 & 105.24 & 105.81 & 104.93 & 1302.42 & 1124.50 & 1193.95 & 1238.61 & 1214.87 & 127.77 & 121.55 & 122.89 & 124.42 & 124.15 \\
\hline & \multicolumn{15}{|c|}{$\begin{array}{l}\text { L.S.D }(0.05) \\
\end{array}$} \\
\hline $\mathbf{F}$ & \multirow{2}{*}{\multicolumn{5}{|c|}{$\begin{array}{l}1.12 \\
0.96\end{array}$}} & \multicolumn{5}{|c|}{78.83} & \multirow{2}{*}{\multicolumn{5}{|c|}{3.01}} \\
\hline I & \multirow{2}{*}{\multicolumn{5}{|c|}{$\begin{array}{l}0.96 \\
\text { NS }\end{array}$}} & \multirow{2}{*}{\multicolumn{5}{|c|}{$\begin{array}{l}59.30 \\
\mathrm{NS}\end{array}$}} & \multirow{2}{*}{\multicolumn{5}{|c|}{$\begin{array}{l}2.62 \\
\text { NS }\end{array}$}} \\
\hline $\mathbf{F} \times \mathbf{I}$ & & & & & & & & & & & & & & & \\
\hline
\end{tabular}


Middle East J. Agric. Res., 8(4): 1344-1360, 2019

Table 3: Effect of intercropping systems, foliar sprays and their interaction on yield attributes of wheat.

\begin{tabular}{|c|c|c|c|c|c|c|c|c|c|c|c|c|c|c|c|}
\hline \multirow{2}{*}{$\begin{array}{r}\text { Intercropping } \\
\text { systems (I) }\end{array}$} & \multicolumn{5}{|c|}{ Number of grains spike ${ }^{-1}$} & \multicolumn{5}{|c|}{ 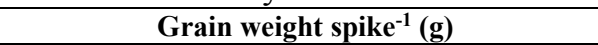 } & \multicolumn{5}{|c|}{1000 grain weight (g) } \\
\hline & $\begin{array}{c}\text { Sole } \\
\text { wheat }\end{array}$ & $\begin{array}{c}\text { Wheat } \\
+ \\
\text { canola }\end{array}$ & $\begin{array}{c}\text { Wheat } \\
+ \\
\text { linseed }\end{array}$ & $\begin{array}{c}\text { Wheat } \\
+ \\
\text { oats } \\
\text { fodder }\end{array}$ & Mean & $\begin{array}{c}\text { Sole } \\
\text { wheat }\end{array}$ & $\begin{array}{c}\text { Wheat } \\
+ \\
\text { canola }\end{array}$ & $\begin{array}{c}\text { Wheat } \\
+ \\
\text { linseed }\end{array}$ & $\begin{array}{c}\text { Wheat } \\
+ \\
\text { oats } \\
\text { fodder }\end{array}$ & Mean & $\begin{array}{c}\text { Sole } \\
\text { wheat }\end{array}$ & $\begin{array}{c}\text { Wheat } \\
+ \\
\text { canola }\end{array}$ & $\begin{array}{c}\text { Wheat } \\
+ \\
\text { linseed }\end{array}$ & $\begin{array}{c}\text { Wheat } \\
+ \\
\text { oats fodder }\end{array}$ & Mean \\
\hline & \multicolumn{15}{|c|}{ Season 2017-18 } \\
\hline Control & 54.53 & 45.80 & 47.13 & 51.53 & 49.75 & 2.21 & 1.78 & 1.91 & 2.03 & 1.98 & 41.63 & 36.20 & 37.60 & 40.03 & 38.87 \\
\hline Salicylic acid & 67.20 & 56.33 & 59.87 & 64.53 & 61.98 & 2.73 & 2.44 & 2.59 & 2.67 & 2.61 & 47.53 & 40.23 & 42.06 & 44.93 & 43.69 \\
\hline Ascorbic acid & 63.20 & 53.93 & 57.00 & 60.06 & 58.55 & 2.61 & 2.09 & 2.15 & 2.47 & 2.33 & 45.40 & 39.63 & 40.20 & 43.70 & 42.23 \\
\hline $\begin{array}{l}\text { Salicylic \& } \\
\text { Ascorbic acid }\end{array}$ & 58.93 & 49.47 & 54.13 & 57.53 & 55.02 & 2.47 & 1.91 & 2.02 & 2.23 & 2.16 & 43.60 & 38.33 & 39.57 & 41.93 & 40.86 \\
\hline Mean & 60.97 & 51.38 & 54.53 & 58.41 & 56.32 & 2.51 & 2.06 & 2.17 & 2.35 & 2.27 & 44.54 & 38.60 & 39.86 & 42.65 & 41.41 \\
\hline & \multicolumn{15}{|c|}{ L.S.D (0.05) } \\
\hline $\mathbf{F}$ & & & 2.97 & & & & & 0.22 & & & & & 1.36 & & \\
\hline I & & & 2.18 & & & & & 0.18 & & & & & 3.30 & & \\
\hline \multirow{2}{*}{$\mathbf{F} \times \mathbf{I}$} & & & NS & & & & & 0.10 & & & & & NS & & \\
\hline & \multicolumn{15}{|c|}{ Season 2018-19 } \\
\hline Control & 53.35 & 45.58 & 47.11 & 51.42 & 49.37 & 2.16 & 1.77 & 1.89 & 2.04 & 1.97 & 41.55 & 35.92 & 37.41 & 39.90 & 38.70 \\
\hline Salicylic acid & 66.83 & 56.00 & 59.29 & 64.73 & 61.71 & 2.72 & 2.42 & 2.58 & 2.67 & 2.60 & 47.30 & 39.93 & 41.93 & 44.87 & 43.51 \\
\hline Ascorbic acid & 62.52 & 53.53 & 56.57 & 59.50 & 58.03 & 2.58 & 2.07 & 2.13 & 2.44 & 2.31 & 45.20 & 39.47 & 39.78 & 43.63 & 42.02 \\
\hline $\begin{array}{l}\text { Salicylic \& } \\
\text { Ascorbic acid }\end{array}$ & 58.08 & 49.07 & 53.57 & 56.90 & 54.41 & 2.45 & 1.89 & 2.03 & 2.21 & 2.15 & 43.47 & 37.90 & 39.31 & 41.78 & 40.62 \\
\hline Mean & 60.20 & 51.05 & 54.14 & 58.14 & 55.88 & 2.48 & 2.04 & 2.16 & 2.34 & 2.25 & 44.38 & 38.31 & 39.61 & 42.55 & 41.21 \\
\hline & \multicolumn{15}{|c|}{ L.S.D (0.05) } \\
\hline $\mathbf{F}$ & & & 2.89 & & & & & 0.22 & & & & & 1.60 & & \\
\hline I & & & 2.22 & & & & & 0.16 & & & & & 3.34 & & \\
\hline $\mathbf{F} \times \mathbf{I}$ & & & NS & & & & & 0.12 & & & & & NS & & \\
\hline
\end{tabular}

NS means not significant. 
Middle East J. Agric. Res., 8(4): 1344-1360, 2019

EISSN: 2706-7955 ISSN: 2077-4605

Table 4: Effect of intercropping systems, foliar sprays and their interaction on biological, grain and straw yields of wheat.

\begin{tabular}{|c|c|c|c|c|c|c|c|c|c|c|c|c|c|c|c|}
\hline \multirow{2}{*}{$\begin{array}{r}\text { Intercropping } \\
\text { systems (I) }\end{array}$} & \multicolumn{5}{|c|}{ Biological yield $\left(\mathrm{kg} \mathrm{ha}^{-1}\right)$} & \multicolumn{5}{|c|}{ Grain yield $\left(\mathrm{kg} \mathrm{ha}^{-1}\right)$} & \multicolumn{5}{|c|}{ Straw yield $\left(\mathrm{kg} \mathrm{ha}^{-1}\right)$} \\
\hline & $\begin{array}{c}\text { Sole } \\
\text { wheat }\end{array}$ & $\begin{array}{c}\text { Wheat } \\
+ \\
\text { canola }\end{array}$ & $\begin{array}{l}\text { Wheat } \\
\text { + linseed }\end{array}$ & $\begin{array}{c}\text { Wheat } \\
+ \\
\text { oats } \\
\text { fodder }\end{array}$ & Mean & $\begin{array}{c}\text { Sole } \\
\text { wheat }\end{array}$ & $\begin{array}{c}\text { Wheat }+ \\
\text { canola }\end{array}$ & $\begin{array}{c}\text { Wheat + } \\
\text { linseed }\end{array}$ & $\begin{array}{c}\text { Wheat } \\
+ \\
\text { oats } \\
\text { fodder }\end{array}$ & Mean & $\begin{array}{c}\text { Sole } \\
\text { wheat }\end{array}$ & $\begin{array}{c}\text { Wheat } \\
+ \\
\text { canola }\end{array}$ & $\begin{array}{l}\text { Wheat } \\
\text { + linseed }\end{array}$ & $\begin{array}{l}\text { Wheat + } \\
\text { oats } \\
\text { fodder }\end{array}$ & Mean \\
\hline & \multicolumn{15}{|c|}{ Season 2017-18 } \\
\hline Control & 13893.00 & 11028.81 & 12510.29 & 13333.33 & 12691.36 & 4920.16 & 3596.71 & 4390.12 & 4587.65 & 4373.66 & 8972.84 & 7432.10 & 8120.17 & 8745.68 & 8317.70 \\
\hline Salicylic acid & 15884.77 & 13086.42 & 14238.68 & 14979.42 & 14547.32 & 5443.62 & 4345.68 & 4864.20 & 5163.79 & 4954.32 & 10441.15 & 8740.74 & 9374.48 & 9815.63 & 9593.00 \\
\hline Ascorbic acid & 15637.86 & 12751.85 & 13991.77 & 14567.90 & 14237.35 & 5359.67 & 4241.09 & 4758.85 & 4888.89 & 4812.13 & 10278.19 & 8510.76 & 9232.92 & 9679.01 & 9425.22 \\
\hline $\begin{array}{l}\text { Salicylic \& Ascorbic } \\
\text { acid }\end{array}$ & 14485.60 & 12363.37 & 13399.18 & 14156.38 & 13601.13 & 5111.11 & 4144.41 & 4669.96 & 4801.65 & 4681.78 & 9374.49 & 8218.96 & 8729.22 & 9354.73 & 8919.35 \\
\hline \multirow[t]{2}{*}{ Mean } & 14975.31 & 12307.61 & 13534.98 & 14259.26 & 13769.29 & 5208.64 & 4081.97 & 4670.78 & 4860.50 & 4705.47 & 9766.67 & 8225.64 & 8864.20 & 9398.76 & 9063.82 \\
\hline & \multicolumn{15}{|c|}{$\begin{array}{l}\text { L.S.D }(0.05) \\
\end{array}$} \\
\hline $\mathbf{F}$ & & & 246.51 & & & & & 172.74 & & & & & 339.58 & & \\
\hline I & & & 805.17 & & & & & 472.65 & & & & & 686.08 & & \\
\hline \multirow{2}{*}{$\mathbf{F} \times \mathbf{I}$} & & & NS & & & & & NS & & & & & NS & & \\
\hline & \multicolumn{15}{|c|}{ Season 2018-19 } \\
\hline Control & 13773.33 & 11036.33 & 12331.00 & 13154.33 & 12573.75 & 5096.57 & 3500.69 & 4246.91 & 4956.65 & 4450.21 & 8676.76 & 7535.64 & 8084.09 & 8197.68 & 8123.54 \\
\hline Salicylic acid & 15664.67 & 12922.00 & 14013.00 & 14748.67 & 14337.09 & 5220.71 & 3871.60 & 4532.37 & 5161.87 & 4696.64 & 10443.96 & 9050.40 & 9480.63 & 9586.80 & 9640.45 \\
\hline Ascorbic acid & 15301.00 & 12650.33 & 13779.33 & 14417.00 & 14036.92 & 5181.62 & 3750.34 & 4473.25 & 5056.24 & 4615.36 & 10119.38 & 8899.99 & 9306.08 & 9360.76 & 9421.55 \\
\hline $\begin{array}{l}\text { Salicylic \& Ascorbic } \\
\text { acid }\end{array}$ & 14186.67 & 12271.67 & 13375.67 & 14035.00 & 13467.25 & 5125.24 & 3537.59 & 4395.06 & 4906.17 & 4491.02 & 9061.43 & 8734.08 & 8980.61 & 9128.83 & 8976.24 \\
\hline \multirow[t]{2}{*}{ Mean } & 14731.42 & 12220.08 & 13374.75 & 14088.75 & 13603.75 & 5156.04 & 3665.06 & 4411.90 & 5020.23 & 4563.31 & 9575.38 & 8555.03 & 8962.85 & 9068.52 & 9040.45 \\
\hline & \multicolumn{15}{|c|}{$\begin{array}{l}\text { L.S.D (0.05) } \\
\end{array}$} \\
\hline $\mathbf{F}$ & \multirow{2}{*}{\multicolumn{5}{|c|}{$\begin{array}{l}384.51 \\
804.37\end{array}$}} & \multicolumn{4}{|c|}{ NS } & \multicolumn{6}{|c|}{437.72} \\
\hline I & & & & & & \multirow{2}{*}{\multicolumn{4}{|c|}{$\begin{array}{l}393.40 \\
\text { NS }\end{array}$}} & \multirow{2}{*}{\multicolumn{6}{|c|}{$\begin{array}{l}\text { NS } \\
\text { NS } \\
\end{array}$}} \\
\hline $\mathbf{F} \times \mathbf{I}$ & \multicolumn{3}{|c|}{ NS } & & & & & & & & & & & & \\
\hline
\end{tabular}


attributes of wheat were found in wheat + oats fodder, i.e plant height $(106.04$ and $124.63 \mathrm{~cm})$, dry matter accumulation (1238.12 and $\left.1238.61 \mathrm{~g} \mathrm{~m}^{-2}\right)$, number. of spike $\mathrm{m}^{-1}(105.81$ and 124.42), number of grains spike ${ }^{-1}$ (58.41 and 58.14), grain weight $\operatorname{spike}^{-1}$ (2.35 and $2.34 \mathrm{~g}$ ), 1000 grain weight (42.65 and $42.55 \mathrm{~g}$ ). Here, the maximum biological, grain, and straw yields of wheat were 14259.26, 4860.50 and $9398.76 \mathrm{~kg} \mathrm{ha}^{-1}$ respectively were produced from wheat + oats fodder in 2017/2018. The corresponding means in 2018-2019 were $14088.75,5020.23$ and $9068.52 \mathrm{~kg} \mathrm{ha}^{-1}$ in the same order. Significantly lowest yield and its attributes were observed in wheat + canola intercropping system than the rest of the intercropping systems. Canola was found to be more aggressive and competitive to wheat than the other crops in intercropping systems. Intercrops canola in wheat was more exposed to the sun and wheat suffered more as it was growing under the canola canopy. Pandey et al., (2015) reported a decrease in performance of wheat in intercropping with rapeseed and mustard. Similar conclusions were reported by and Said and Hamd-Alla (2018) and Hamada and Hamd-Alla (2019).

\section{Effect of foliar sprays on yield and its attributes.}

The data showed that foliar sprays affected significantly all studied traits except grain yield in the second season. Values from Table 1, 2, 3, and 4 indicated that among the foliar sprays higher values of yield and its attributes was found in the salicylic acid (SA) and ascorbic acid (AA) @ 200 ppm as compared with another treatment. The SA produced the maximum values of all traits such as leaf area (2.69 and 2.64), PAR interception (74.03 and 73.75 per cent) and chlorophyll index (55.24 and 55.43) in the first and second seasons, respectively at 120 DAS. Here too, the data emphasized that average yield attributes viz. plant height $(108.03$ and $107.90 \mathrm{~cm})$, dry matter accumulation (1294.01 and $1290.69 \mathrm{~g} \mathrm{~m}^{-2}$ ), number. of spike $\mathrm{m}^{-1}$ (128.56 and 128.13), number of grains spike ${ }^{-1}$ (61.98 and 61.71), grain weight spike ${ }^{-1}(2.61$ and $2.60 \mathrm{~g}), 1000$ grain weight (43.69 and $43.51 \mathrm{~g}$ ). The average biological, grain and straw yields of wheat obtained were $14547.32,4954.32$ and $9593.00 \mathrm{~kg}$ $\mathrm{ha}^{-1}$ respectively, were obtained from salicylic acid (SA) @ $200 \mathrm{ppm}$ in the first season. The corresponding means in the second season were $14337.09,4696.64$ and $9640.45 \mathrm{~kg} \mathrm{ha}^{-1}$ in the same order. The present trend is can expected for three reasons. The first is due to the fact that salicylic acid plays as a signal in plant-microbe interaction and antioxidants which are considered safe to human and environment had been used successfully to control some plant diseases. The second reason is the assigned diverse regulatory roles of SA in the metabolism of plants, it is a natural product of phenylpropanoid metabolism. The third reason is salicylic acid has direct involvement in plant growth thermogenesis, flower induction and uptake of ions. The present results are in accordance with those obtained by previous authors (Elad 1992, Popova et al., 1997, Galal et al., 2000, Abdel Ati et al., 2000, Bakry et al., 2013, Farhadi, et al., 2017, Noreen et al., 2017, Ahmad et al., 2018 and Said and Hamd-Alla 2018).

\section{Effect of the interaction between intercropping systems and foliar sprays on yield and its attributes.}

The data recorded in Table 1, 2, 3, and 4 showed that the interaction between intercropping system and foliar sprays could not exhibit any significant differences in all traits except of dry matter accumulation $\mathrm{g} \mathrm{m}^{-2}$ in the first season only, as well as grain weight spike ${ }^{-1}$ in both the seasons. The highest mean value of dry matter accumulation was obtained with sole wheat under salicylic acid (SA) @ 200 ppm (1414.56 $\left.\mathrm{g} \mathrm{m}^{-2}\right)$. Meanwhile, the highest grain weight spike ${ }^{-1}$ (2.73 and 2.72) in the first and second seasons, respectively was produced from sole wheat with $200 \mathrm{ppm}$ of salicylic acid. This may be attributed to the fact that SA enhances growth vigor of plants and increased plant growth due to exogenous application of salicylic acid may be contributed in physiological processes, such as stomatal conductance, minerals uptake, membrane activity and photosynthesis hence affected growth. The present results confirmed with those obtained by previous authors (Zilic et al., 2010 Said and Abd El-Moneem 2016, Dragicevic et al., 2017 and Said and Hamd-Alla 2018).

\section{II- Oats fodder}

The data presented in Table $5 \& 6$ indicated that fresh forage yield of oats fodder was affected significantly by intercropping systems and foliar sprays in both seasons. Sole oats fodder had the highest value of fresh forage in the first and cut were 15354.9 and $16077 \mathrm{~kg} \mathrm{ha}^{-1}$ as compared with 
intercrop 4197.7 and $4433 \mathrm{~kg} \mathrm{ha}^{-1}$ in the 2017-18 and 2018-19 seasons, respectively. While in the second cut 29282.41 and $29607.89 \mathrm{~kg} \mathrm{ha}^{-1}$ as compared with intercrop 14835.39 and $14871.11 \mathrm{~kg} \mathrm{ha}^{-1}$ in the first and second season, respectively for sole oats fodder as compared to intercrops oats fodder in wheat in the second cuts were for. The salicylic acid produced the maximum value 23618.83 and $23491.32 \mathrm{~kg} \mathrm{ha}^{-1}$, in the first and second seasons, respectively. Control produced the lowest values of 20403.81 and $20896.59 \mathrm{~kg} \mathrm{ha}^{-1}$ in 2017-18 and 2018-19 seasons, respectively. Fresh forage yield was more in sole planting as compared to the intercropped oats fodder, it may be due to the fact that oats fodder was sown in the furrow and wheat was sown on the beds which results the poor light interception to the component crops. These results are in conformity with (Khatun et al., 2012, and Sebetho et al., 2014) Hamd-Alla et al., (2014) also reported sole cowpea had the highest values of fresh forage as compared to their intercropping systems. On the contrary, Dube et al., 2014 and Matusso et al., (2014) showed that intercropping patterns recorded the highest fresh weight.

Table 5: Effect of intercropping systems on fresh forage yield of intercrops oats fodder in wheat in $\mathrm{I}^{\mathrm{st}}$ cut.

\begin{tabular}{|c|c|c|}
\hline Treatment & & Fresh forage yield $\left(\mathrm{kg} \mathrm{ha}^{-1}\right)$ \\
\hline & Season 2017-18 & \\
\hline Sole & & 15354.9 \\
\hline Intercrop & & 4197.7 \\
\hline F Test & & $* *$ \\
\hline & Season 2018-19 & \\
\hline Sole & & 16077 \\
\hline Intercrop & & 4433 \\
\hline F Test & & $*$ \\
\hline
\end{tabular}

Table 6: Effect of intercropping systems, foliar sprays and their interaction on fresh forage yield of intercrops oats fodder in wheat in $2^{\text {nd }}$ cut.

\begin{tabular}{|c|c|c|c|}
\hline \multirow[b]{2}{*}{ Foliar sprays (F) } & \multicolumn{3}{|c|}{ Fresh forage yield $\left(\mathrm{kg} \mathrm{ha}^{-1}\right)$} \\
\hline & Sole & Intercrop & Mean \\
\hline \multicolumn{4}{|c|}{ Season 2017-18 } \\
\hline Control & 27391.98 & 13415.64 & 20403.81 \\
\hline Salicylic acid & 30941.36 & 16296.30 & 23618.83 \\
\hline Ascorbic acid & 29861.11 & 15308.64 & 22584.88 \\
\hline Salicylic \& Ascorbic acid & 28935.19 & 14320.99 & 21628.09 \\
\hline Mean & 29282.41 & 14835.39 & 22058.90 \\
\hline L.S.D (0.05) (F) & & 653.75 & \\
\hline F test & & $* *$ & \\
\hline $\mathbf{F} \times \mathbf{I}$ & & NS & \\
\hline \multicolumn{4}{|c|}{ Season 2018-19 } \\
\hline Control & 28244.86 & 13548.33 & 20896.59 \\
\hline Salicylic acid & 30845.14 & 16137.50 & 23491.32 \\
\hline Ascorbic acid & 30080.25 & 15363.89 & 22722.07 \\
\hline Salicylic \& Ascorbic acid & 29261.32 & 14434.72 & 21848.02 \\
\hline Mean & 29607.89 & 14871.11 & 22239.50 \\
\hline L.S.D (0.05) (F) & & 1111.4 & \\
\hline F test & & $* *$ & \\
\hline $\mathbf{F} \times \mathbf{I}$ & 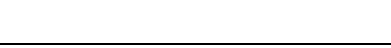 & NS & \\
\hline
\end{tabular}

\section{III- Canola}

The results reported in Table 7 showed that intercropping system and foliar sprays of affected significantly on seed yield in the two growing seasons. Sole canola produced the maximum values of seed yield as compared to intercrop canola in wheat in both seasons. The salicylic acid (SA) @ 200 ppm produced the maximum values of seed yield 1295.28 and $1422.73 \mathrm{~kg} \mathrm{ha}^{-1}$ in 2017-18 and 201819 seasons, respectively. The lowest value in this respect was obtained from control 1070.01 and $1279.15 \mathrm{~kg} \mathrm{ha}^{-1}$ in the first and second seasons, respectively. 


\section{IV- Linseed}

Data illustrated in Table 7 focuses that intercropping system and foliar sprays and affected significantly $(\mathrm{p} \leq 0.05)$ on seed yield in both seasons. The maximum value of yield 1542.89 and $1572.14 \mathrm{~kg} \mathrm{ha}^{-1}$ was obtained from sole linseed compared to intercrop linseed in wheat 285.24 and $376.00 \mathrm{~kg} \mathrm{ha}^{-1}$ in the first and second seasons, respectively. The salicylic acid @, 200 gave the highest values in both seasons. The control gave the lowest values in this respect. These crops possessed different nature of growth, growth duration and plant habit, rooting pattern, canopy structure and days to maturity. The linseed, which was of long duration, possessed larger plant canopy and slower growth rate, during initial growth. This was the reason that component crops respond differentially in intercropping system and foliar sprays.

The canola and linseed produced higher seed yield in the second season as compared to the first season. It was due to more precipitation received during the crop season in 2017-18 than during 201819. The average temperature during February to April coinciding with the reproductive and maturity stages of the wheat, canola and linseed crops remained milder during second year than the first. This elongated the growing periods of these crops during second seasons than the first and resulted in a slightly better performance of wheat, canola and linseed. Yield potential of the intercrops was comparatively higher in oats fodder and canola as compared to linseed in intercropping system irrespective of their sole planting. Similar results in this respect were observed by previous authors (Pandey a et al., 2015, Said and Abd El-Moneem 2016, Dragicevic et al., 2017 and Said and HamdAlla 2018).

Table 7: Effect of intercropping systems, foliar sprays and their interaction on grain yields of intercrops canola and linseed in wheat.

\begin{tabular}{|c|c|c|c|c|c|c|}
\hline \multirow{2}{*}{$\begin{array}{l}\text { Intercropping systems (I) } \\
\text { Foliar sprays (F) }\end{array}$} & \multicolumn{3}{|c|}{ Seed canola $\left(\mathrm{kg} \mathrm{ha}^{-1}\right)$} & \multicolumn{3}{|c|}{ Seed linseed $\left(\mathrm{kg} \mathrm{ha}^{-1}\right)$} \\
\hline & Sole & Intercrop & Mean & Sole & Intercrop & Mean \\
\hline \multicolumn{7}{|c|}{ Season 2017-18 } \\
\hline Control & 1754.21 & 385.80 & 1070.01 & 1365.63 & 233.54 & 799.59 \\
\hline Salicylic acid & 1952.69 & 637.86 & 1295.28 & 1663.70 & 363.17 & 1013.44 \\
\hline Ascorbic acid & 1973.91 & 554.53 & 1264.22 & 1622.22 & 287.04 & 954.63 \\
\hline Salicylic \& Ascorbic acid & 1869.53 & 432.10 & 1150.82 & 1520.00 & 257.20 & 888.60 \\
\hline Mean & 1887.59 & 502.57 & 1195.08 & 1542.89 & 285.24 & 914.06 \\
\hline L.S.D (0.05) (F) & & 144.65 & & & 111.8 & \\
\hline F test & & $* *$ & & & $* *$ & \\
\hline $\mathbf{F} \times \mathbf{I}$ & & NS & & & NS & \\
\hline \multicolumn{7}{|c|}{ Season 2018-19 } \\
\hline Control & 1863.15 & 695.14 & 1279.15 & 1483.33 & 350.75 & 917.04 \\
\hline Salicylic acid & 2055.89 & 789.57 & 1422.73 & 1627.05 & 398.22 & 1012.64 \\
\hline Ascorbic acid & 2001.54 & 718.37 & 1359.96 & 1599.52 & 383.37 & 991.45 \\
\hline Salicylic \& Ascorbic acid & 1901.57 & 709.63 & 1305.60 & 1578.66 & 371.67 & 975.17 \\
\hline Mean & 1955.54 & 728.18 & 1341.86 & 1572.14 & 376.00 & 974.07 \\
\hline (F) L.S.D (0.05) & & NS & & & NS & \\
\hline (I) $F$ test & & ** & & & ** & \\
\hline$F \times I$ & & NS & & & NS & \\
\hline
\end{tabular}

NS and ${ }^{* *}$ means not significant and significant at 0.01 probability, respectively.

\section{Intercropping indices}

\section{Land equivalent ratio (LER)}

The data on LER of different intercropping systems presented in Table 8 indicate that LER values were greater than one in all the intercropping treatments expect that of canola in the first season. LER indicated the yield advantage over sole crops due to the better utilization of environmental resources for growth in both seasons. LER of wheat was low in wheat + canola intercropping system than the other intercropping systems in both the growing seasons. The salicylic acid and wheat + oats fodder gave higher LER (1.48 and 1.51) in first and second seasons, respectively compared with the other treatments. It may be attributed to the fact that canola is more aggressive and dominant than rest of the intercrops, i.e. linseed and oats fodder. All these intercrops 
Table 8: Effect of intercropping systems and foliar sprays on land equivalent ratio (LER) and competitive ratio (CR) of wheat based intercropping system.

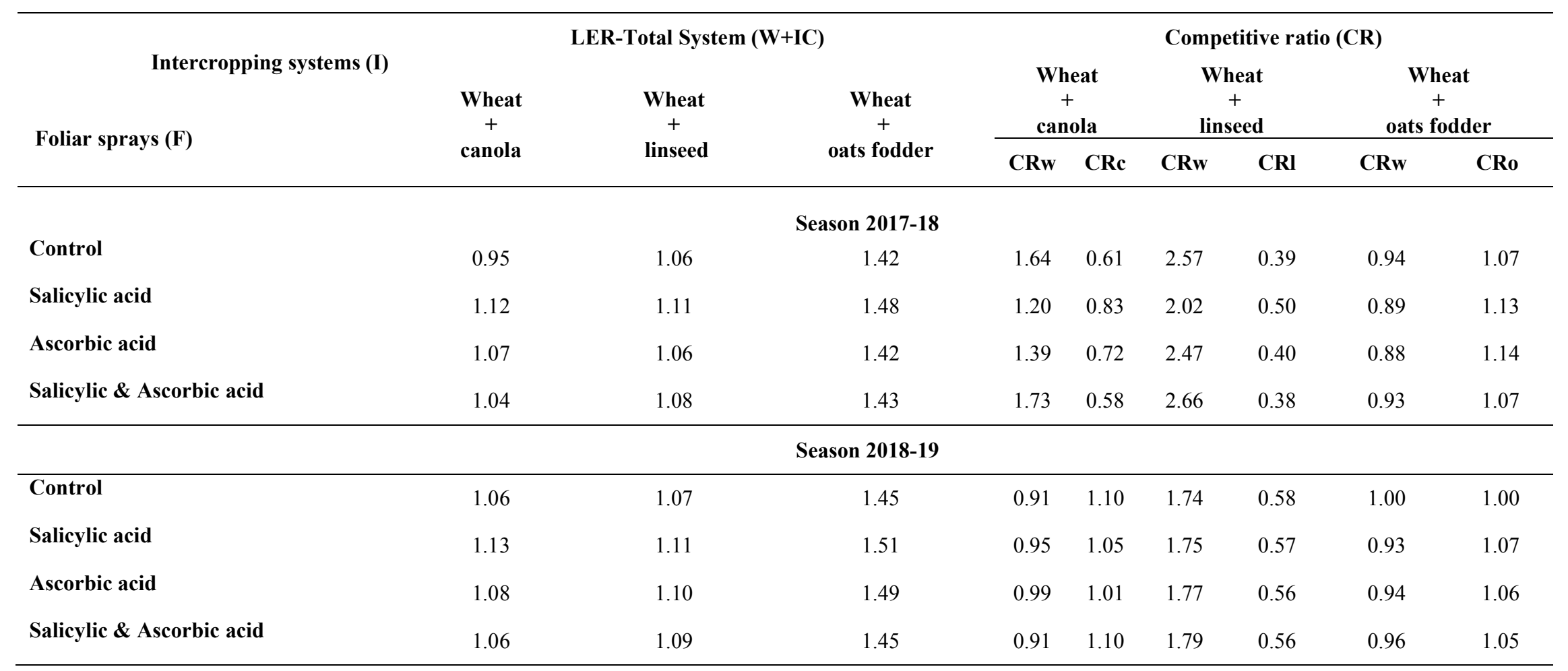


possess different nature of growth, duration, plant habit, rooting pattern, canopy structure and days to maturity. Therefore, these crops differ in yield potential and possess differential competitive ability. Value of LER of intercrops was higher in oats fodder, followed by linseed $>$ canola in both seasons. Oats fodder is a fast growing crop, having higher yield potential, short duration and less competitive as compared to canola and linseed.

\section{Competitive ratio (CR)}

Competitive ratio (CR) is another way to know the degree with which one crop competes with another component crop. The data regarding (CR) value are presented in Table 8. The salicylic \& ascorbic acid and wheat + linseed gave higher $\mathrm{CR}$ values (2.66 and 1.79) in first and second seasons, respectively compared with the other treatments. The results of (CR) values showed that wheat was the dominant species with higher values over the intercrops in the intercropping systems. CR values for wheat was higher in wheat + linseed followed by wheat + canola $>$ wheat + oats fodder intercropping systems. It indicated that wheat was more competitive to the linseed, whereas wheat was least competitive to canola and oats fodder in the intercropping system in both the seasons.

\section{Monetary advantage Index (MAI)}

Monetary advantage index (MAI) values presented in Table 9 were positive in both the intercropping systems and foliar sprays implying a definite yield advantage in both the seasons except MAI index of canola in the first seasons. MAI values (446.00 and 464.19) were higher in salicylic acid with wheat + oats fodder than other treatments in the first and second seasons. MAI index values showed the economic advantage of salicylic acid as compared to control. MAI index values were higher in wheat + oats fodder intercropping system followed by wheat + linseed $>$ wheat + canola because intercrops were differing in competitive ability and in yield potential in the intercropping system. Wheat + oats fodder intercropping systems gave the higher yield advantage than the other intercropping system in both the yield advantage indices. Monetary advantage index (MAI) values are based on land equivalent ratio (LER). As reported by other studies (Ghosh, 2004, Dube et al., 2014, Said and Hamd-Alla 2018, Hamada and Hamd-Alla 2019 and Nagwa R. Ahmed et al., 2019).

Table 9: Effect of intercropping systems and foliar sprays on monetary advantage index (MAI) of wheat based intercropping system.

\begin{tabular}{|c|c|c|c|}
\hline \multirow{2}{*}{$\begin{array}{l}\text { Intercropping systems (I) } \\
\text { Foliar sprays (F) }\end{array}$} & \multicolumn{3}{|c|}{ Monetary advantage index (MAI) } \\
\hline & Wheat + canola & Wheat + linseed & Wheat + oats fodder \\
\hline \multicolumn{4}{|c|}{ Season 2017-18 } \\
\hline Salicylic acid & 161.59 & 152.25 & 446.00 \\
\hline Ascorbic acid & 93.65 & 82.19 & 385.02 \\
\hline Control & 74.85 & 89.40 & 408.36 \\
\hline Salicylic acid & 169.67 & 146.30 & 464.19 \\
\hline Ascorbic acid & 103.78 & 131.80 & 442.43 \\
\hline Salicylic \& Ascorbic acid & 75.88 & 117.31 & 404.84 \\
\hline
\end{tabular}

\section{Wheat equivalent yield}

The data in Table 10 showed that wheat equivalent yield was higher in salicylic acid with wheat + oats fodder 6338.70 and $6389.72 \mathrm{~kg} \mathrm{ha}^{-1}$ ) as compared to control with wheat + canola (4487.31 and $5087.42 \mathrm{~kg} \mathrm{ha}^{-1}$ ) in the first and the second seasons, respectively. The wheat equivalent yield of salicylic acid with wheat + linseed was 5910.37 and $5679.42 \mathrm{~kg} \mathrm{ha}^{-1}$ in the first and second seasons, respectively. All the component crops (oats fodder, canola and linseed) in intercropping system with wheat resulted in the higher values of wheat equivalent yield than their respective sole planting except sole crop of canola in both the seasons. Wheat + oats fodder intercropping system produced the higher wheat equivalent yield than the others wheat based intercropping systems and their respective sole crops of wheat and the component crops. Oats fodder is a fast growing crop, having higher yield potential, short duration and less competitive as compared to canola and linseed. 
intercropping system which was numerically higher but statistically at par with the sole bed planted wheat. (Khatun et al., 2012) also fawned that all the intercropping treatments gave higher wheat equivalent yield than their respective sole crops.

Table 10: Effect of intercropping systems and foliar sprays on wheat equivalent yield.

\begin{tabular}{|c|c|c|c|c|c|c|c|}
\hline $\begin{array}{r}\text { Intercropping } \\
\text { systems (I) } \\
\text { Foliar sprays (F) }\end{array}$ & $\begin{array}{c}\text { Sole } \\
\text { wheat }\end{array}$ & $\begin{array}{c}\text { Sole } \\
\text { canola }\end{array}$ & $\begin{array}{c}\text { Wheat }+ \\
\text { canola }\end{array}$ & $\begin{array}{c}\text { Sole } \\
\text { linseed }\end{array}$ & $\begin{array}{c}\text { Wheat + } \\
\text { linseed }\end{array}$ & $\begin{array}{c}\text { Sole oats } \\
\text { fodder }\end{array}$ & $\begin{array}{c}\text { Wheat }+ \text { oats } \\
\text { fodder }\end{array}$ \\
\hline \multicolumn{8}{|c|}{ Season 2017-18 } \\
\hline Salicylic acid & 5444.30 & 4453.64 & 5815.28 & 4786.34 & 5910.37 & 2236.52 & 6338.70 \\
\hline Ascorbic acid & 5360.23 & 4502.03 & 5521.00 & 4667.00 & 5586.14 & 2158.44 & 5992.12 \\
\hline \multicolumn{8}{|c|}{ Season 2018-19 } \\
\hline Control & 5096.57 & 4252.84 & 5087.42 & 4272.64 & 5257.22 & 2149.07 & 5987.50 \\
\hline Salicylic acid & 5220.71 & 4692.79 & 5673.88 & 4686.61 & 5679.42 & 2346.91 & 6389.72 \\
\hline Ascorbic acid & 5181.62 & 4568.73 & 5390.10 & 4607.31 & 5577.52 & 2288.71 & 6225.23 \\
\hline $\begin{array}{l}\text { Salicylic \& Ascorbic } \\
\text { acid }\end{array}$ & 5125.24 & 4340.54 & 5157.40 & 4547.23 & 5465.63 & 2226.40 & 6004.46 \\
\hline
\end{tabular}

\section{Conclusion}

As evident by the results, it could be recommended that that the highest mean values of growth, yields and its attributes which were obtained from wheat + oats fodder significantly higher than wheat + linseed and wheat + canola intercropping systems in both the seasons. The salicylic@200 ppm produced the maximum values for all the traits as compared to the control in the two growing seasons. Land equivalent ratio indicated the yield advantage over sole crops. Competitive ratio showed that wheat was the dominant species with the higher values over the intercrops in the intercropping systems. Monetary advantage index values showed the economic advantage in salicylic acid@200 with wheat + oats fodder as compared to other treatments. All the component crops (oats fodder, canola and linseed) in intercropping system with wheat resulted in the higher value of wheat equivalent yield than their respective sole planting except sole crop of canola in both the seasons.

\section{Acknowledgments}

Grateful appreciation to Department of Crop Intensification Research, Field Crops Research Institute, Agricultural Research Center, Egypt, for their valuable help and encouragement. The authors greatly acknowledge the Department of Science and Technology (DST) India, and Department of Agronomy, Punjab Agricultural University, Ludhiana, for their assistance to conduct this study for providing us field and laboratory facilities. The authors gratefully thank the staff of Agronomy Department Farm at Punjab Agricultural University for their excellent technical assistance.

\section{References}

Abdel Ati, Y.Y., S.H. Gad El-Hak, A.A. Galal and Y.M.M. Moustafa, 2000. Effect of some antioxidant compounds on some horticultural characters of four new F1 hybrids of tomato. J. Agric. Sci. Mansoura Univ., 25: 1673-1692.

Ahmad H., I. Khan, W. Liaqat, M. F. Jan and M. D. Ahmadzai, 2018. Effect of salicylic acid on yield and yield components of maize under reduced irrigation. Int J Environ Sci Nat Res., 9 (3): 1-5.

Anjaneyulu V R, S. P. Singh and M. Pal, 1982. Effect of competition free period and technique and pattern of pearl millet planting for growth and yield of mungbean and total productivity in soil for pearl millet and pearl millet/ mungbean intercropping system. Indian J. Agron 27: 219-26.

Bakry, B.A., T.A. Elewa, M.F. El-Kramany and A.M. Wali, 2013. Effect of humic and ascorbic acids foliar application on yield and yield components of two Wheat cultivars grown under newly reclaimed sandy soil. Int. J. Agron. Plant Prod., 4: 1125-1133.

Dhima, K.U., A.A. Lithourgidis, I.B. Vasilakoqlou and C.A. Dordas, 2007. Competition indices of common vetch and cereals intercropping in two seeding ratio. Field Crops Res., 100: 249-258. 
Doaeres, S.H., J. Narvasez-Vasquez, A. Conconi and C.A. Ryan, 1995. Salicylic acid inhibits synthesis of proteinase inhibitors in tomato leaves induced by system in and jasmonic acid. Plant Physiology, 108: 1741-1746.

Dragicevic V.D., S.I. Oljaca, M.S. Simic, Z.K. Dolijanovic, B.J. Kresovic and M.Z. Brankov, 2017. Content of some antioxidants in intercropped maize and soybean grain. J. of Agric. Sci., 62 (1): 31-40.

Dube, E.D.N., T. Madanzi1, A. Kapenzi and E. Masvaya, 2014. Root length density in maize/cowpea intercropping under a basin tillage system in a semi-arid area of Zimbabwe. American Journal of Plant Sciences, 5: 1499-1507.

Elad, Y., 1992. The use of antioxidants (free radical scavengers) to control gray mould (Botrytis cinerea) and white mould (Sclerotinia sclerotiorum) in various crops. Plant Pathology, 41: 417426.

Farhadi, N., M. Sayyahfar and G. Shakarami, 2017. Effect of foliar applications of various levels of salicylic acid on yield and yield components of two barley cultivars under dry land farming conditions. J. Res. Agric., 8 (5): 25-40.

Fercha, A., G. Hocine and B. Mebarek, 2011. Improvement of salt tolerance in durum wheat by ascorbic acid application. J. Stress Physiol. Biochem., 7: 27-37.

Galal, A.A., S.H. Gad El-Hak, Y.Y. Abdel-Ati and Y.M.M. Moustafa, 2000. Response of new tomato hybrids to some antioxidants and early blight. The $2^{\text {nd }}$ Scientific Conference of Agricultural Sciences, Assuit, Egypt, pp: 673-686.

Ghosh P K., 2004. Growth, yield, competition and economics of groundnut/ cereal fodder intercropping systems in the semi- arid tropics of India. Field Crops Res 88: 227-37.

Hamada A. and W. A. Hamd Alla, 2019. Productivity of intercropped wheat with faba bean under crop sequences and foliar application of humic Egypt. J. Agron. 41 (3): 225-241.

Hamd Alla, W. A., E. M. Shalaby, R. A. Dawood and A. A. Zohry, 2014. Effect of cowpea (Vigna sinensis L.) with maize (Zea mays L.) intercropping on yield and its components. World Academy of Science, Engineering and Technology Inter. J. of Biolog. Veter. Agric. and Food Engin. 8 (11): 1170-1176.

Hussain, M.L., S.H. Shah, S., Hussain and K. Iqbal, 2002. Growth, yield and quality response of three wheat (Triticum aestivum L.) varieties to different levels of N, P and K. International Journal of Agriculture and Biology 4:189-198.

Hussein, M.M. and A.K. Alva, 2014. Effects of zinc and ascorbic acid application on the growth and photosynthetic pigments of millet plants grown under different salinity. Agric. Sci., 5: 12531260 .

Khatun S, A. K. Azad and P. Bala, 2012. Intercropping with wheat affected crop productivity. Bangladesh Res J., 6: 414-19.

Matusso, J.M.M, J.N. Mugwe and M. Mucheru-Muna, 2014. Effects of different maize (Zea mays L.)-Soybean (Glycine max L. Merrill) intercropping patterns on yields and its economics. Academia Journal of Agricultural Research. 2(7): 159-166.

Mead R. and R W., Willey, 1980. The concept of a 'land equivalent ratio' and advantages in yields from intercropping. Experimental Agric 16: 217-28.

Nagwa R.A, A.M. Sheha and W.A. Hamd-All, 2019. Effect of intercropping of some legume forage crops with maize under levels of mineral NPK and nano NPK fertilizer. J. of Plant Production, Mansoura Univ., 10(10):833-842.

Noreen, S., K. Fatima, H.U.R. Athar, S. Ahmad and K. Hussain, 2017. Enhancement of physiobiochemical parameters of wheat through exogenous application of salicylic acid under drought stress. The J. Anim. Plant Sci., 27(1): 153- 163.

Pandey M., T. Singh and S. Singh, 2015. Effect of Row Orientation and Intercropping on Dry Matter Accumulation and Growth Parameters of Bed Planted Wheat (Triticum aestivum L.). Inter., J., of Agric., Scie., 7(11): 757-761.

Popova, L., T. Pancheva and A. Uzunova, 1997. Salicylic acid: Properties, biosynthesis and physiological role. Bulg. J. Plant Physiol., 23: 85-93.

Said, M.T. and A.M.A. Abd El-Moneem, 2016. Response of two bread wheat cultivars to foliar spray by salicylic and ascorbic acids under water stress conditions. Assiut J. Agric. Sci., 47: (6-2): 391-404. 
Said, M. T. and W. A. Hamd-Alla, 2018. Impact of foliar spraying with antioxidant and intercropping pattern of maize and soybean on yields and its attributes. J. Plant Prod., Mansoura Univ., 9 (12): 1069-1073.

SAS Institute, 2008. The SAS system for windows, release 9.2. Cary NC: SAS Institute.

Sebetho, E.T., AT. Modi and LG. Owoeye, 2014. Maize-cowpea rotation and intercropping effects on cowpea yield. Ph. D. Thesis, University of KwaZulu- Natal.

Smirnoff, N. and G.L. Wheeler, 2000. Ascorbic acid in plants. Biosynthesis and Function. Current Review in Plant Sciences, 19: 267-290.

Steel, G. D. and J. H. Torrie, 1981. Principles and Procedures of Statistics (2 ${ }^{\text {nd }}$ edition) McGraw-Hill Book Company. Inc. N. Y. xxi - 633.

Wang, F, Ling'an Kong, Ken Sayre, Shengdong Li, Jisheng Si, Bo Feng and Bin Zhang, 2011. Morphological and yield responses of winter wheat (Triticum aestivum L.) to raised bed planting in Northern China. Afric J Agric Res 6: 2991-97.

Willey, R.W. and M. R. Rao, 1980. Competitive ratio for quantifying competition between intercrops. Exp. Agric. f6, 117-125.

Willey, R.W., 1985. Intercropping its importance and research needs. Part I: Competition and yield advantages. Field Crop Abst. 32: 1-10.

Zilic S., V. h. Sukalovic, V. Maksimovic, M. Maksimović, Z. Basic, Vesna Peric and J. D. Maksimovic, 2010. Antioxidant properties of soybean with black and yellow kernel coat $46^{\text {th }}$ Croatian and $6^{\text {th }}$ International Symposium on Agriculture. Opatija. Croatia., pp: 686-689. 